\title{
Mesh or not to Mesh in Female Pelvic Organ Prolapse: This is the Problem!
}

\author{
Giampiero Capobianco $^{* 1,2}$, Pier Luigi Cherchi ${ }^{1}$ and Salvatore Dessole ${ }^{1}$ \\ ${ }^{1}$ Gynecologic and Obstetric Clinic, Sassari University, Italy \\ ${ }^{2}$ Department of Surgical, Microsurgical and Medical Sciences Viale San Pietro 12,07100,Sassari, Italy
}

Pelvic organ prolapse $[\mathrm{POP}]$ is an anatomic condition that can have a marked effect on quality of life [QoL]. The prevalence increases and the number of prolapse surgeries will have a $47 \%$ annual increase [1]

The high rate $[30 \%]$ of reoperation for recurrence [relapsed POP], after native tissue repair [traditional surgery] and evidence that mesh insertion is an effective treatment for abdominal hernia surgery, favored the introduction of mesh for POP repair.

POP repairs by vaginal approach can be technically difficult. Pelvic surgeons have used mesh to augment advanced POP repairs. Transvaginal mesh repair for POP were introduced to the market in the 1990s as an alternative to traditional native tissue repair and quickly became widely used [2].

Success, that was in the past based on restoration of anatomy, has recently been redefined to include the absence of symptoms and correlation with patient perception of outcomes.

Abdominal sacrocolpopexy with mesh has become the goal standard technique for patients with high-grade vaginal prolapse. However, abdominal approach has been substituted by less invasive laparoscopic and robotic techniques because of the morbidity of the open abdominal approach [3]. Vaginal repair with mesh has the same goal as the abdominal approach but without the morbidity of abdominal surgery [3].

The Cochrane review [4] reports that transvaginal permanent mesh is associated with lower rates of awareness of prolapse, reoperation for prolapse, and prolapse on examination than native tissue repair, but is also associated with higher rates of stress urinary incontinence, or mesh exposure and higher rates of bladder injury at surgery and de novo stress urinary incontinence. The risk-benefit profile means that transvaginal mesh has limited utility in primary surgery. While it is possible that in women with higher risk of recurrence the benefits may outweigh the risks, there is currently no evidence to support this position. Limited evidence suggests that absorbable mesh may reduce rates of recurrent prolapse on examination compared to native tissue repair, but there was insufficient evidence on absorbable mesh to draw any conclusions for other outcomes. There was also insufficient evidence to draw any conclusions regarding biological grafts compared to native tissue repair. In 2011 many transvaginal permanent meshes were voluntarily withdrawn from the market, and the newer, lightweight transvaginal permanent meshes still available have not been evaluated within a randomized controlled trial [RCT] In the meantime, these newer transvaginal meshes should be utilized under the discretion of the ethics committee [4].

The complication rates of mesh seem to differ based on type of procedure, surgeon experience, and patient medical comorbidities [5].

With regards to uterine sparing surgery, high satisfaction and low reoperation rates can be reached using a variety of hysteropexy

\section{Publication History:}

Received: December 27, 2017

Accepted: January 17, 2018

Published: January 19, 2018

\section{Keywords:}

Pelvic organ prolapse, Abdominal sacrocolpopexy, Biological graft Polypropylene mesh, Vaginal repair

techniques [6]. However, according to Cochrane reviews [7] no clear conclusion can be reached from the available data comparing uterine preserving surgery versus vaginal hysterectomy for uterine prolapse.

With regard to anterior compartment prolapse, to reduce the rate of recurrent prolapse after traditional native tissue repair [anterior colporrhaphy], clinicians have used different surgical techniques. According to Cochrane review [8], biological graft repair or absorbable mesh provides minimal advantage compared with native tissue repair. Native tissue repair was associated with increased awareness of prolapse and increased risk of repeat surgery for prolapse and recurrence of anterior compartment prolapse compared with polypropylene mesh repair. However, native tissue repair was associated with reduced risk of de novo stress urinary incontinence (SUI), reduced bladder injury, and reduced rates of repeat surgery for prolapse, SUI and mesh exposure. Current evidence does not support the use of mesh repair compared with native tissue repair for anterior compartment prolapse owing to increased morbidity. Clinicians and women should be cautious when utilizing these products, as their safety and efficacy have not been established [8].

Recently, two parallel-group, multicentre, randomized controlled trials were performed [9] (PROSPECT [PROlapse Surgery: Pragmatic Evaluation and randomised Controlled Trials]) in 35 centres [a mix of secondary and tertiary referral hospitals] in the UK. The authors recruited women undergoing primary transvaginal anterior or posterior compartment prolapse surgery by 65 gynaecological surgeons in these centres. They randomly assigned participants by a remote web based randomisation system to one of the two trials: comparing standard [native tissue] repair alone with standard repair augmented with either synthetic mesh [the mesh trial] or biological graft [the graft trial]. The authors assigned women [1:1:1 or 1:1] within three strata: assigned to one of the three treatment

*Corresponding Author: Prof. Giampiero Capobianco, Department of Surgical, Microsurgical and Medical Sciences Viale San Pietro 12,07100,Sassari, Italy, Tel. +393392897821, Fax +39079228265; E-mail: capobia@uniss.it

Citation: Capobianco G, Luigi P, Dessole S (2018) Mesh or not to mesh in female pelvic organ prolapse: this is the Problem!. Int J Gynecol Clin Pract 2: 137. https:// doi.org/10.15344/2394-4986/2018/137

Copyright: (c) 2018 Capobianco et al. This is an open-access article distributed under the terms of the Creative Commons Attribution License, which permits unrestricted use, distribution, and reproduction in any medium, provided the original author and source are credited. 
options, comparison of standard repair with mesh, and comparison of standard repair with graft. Participants, ward staff, and outcome assessors were masked to randomization where possible; masking was obviously not possible for the surgeon. Follow-up was for 2 years after the surgery; the primary outcomes, measured at 1 year and 2 years, were participant-reported prolapse symptoms (i.e. the Pelvic Organ Prolapse Symptom Score [POP-SS]) and condition specific [ie, prolapse-related] quality-of-life scores, analyzed in the modified intention-to-treat population. The authors reported that augmentation of a vaginal repair with mesh or graft material did not improve women's outcomes in terms of effectiveness, quality of life, adverse effects, or any other outcome in the short term, but more than $10 \%$ women had a mesh complication. Therefore, follow-up is vital to identify any longer-term potential benefits and serious adverse effects of mesh or graft reinforcement in vaginal prolapse surgery [9].

In conclusion, todsay's, the following recommendations, evidence medicine based [EBM], could be used:

1. Fascial surgery has a fundamental role in primary POP surgery.

2. Mesh could be used in prolapse relapses and in POP > III degree.

3. Possible use of mesh in primary surgery only with adequate patient selection after positive opinion of Ethics Committee [4].

4. Synthetic meshes should be preferred.

5. The transobturator [TOT] and/or transperineal [TP] pass meshes should be abolished in the posterior compartment prolapse correction for the high risk of complications.

The destiny of vaginal mesh, such as its effects on sexual function, the potential for urinary tract erosion or vaginal extrusion and the possibility of pelvic pain related to the mesh, should be considered and balanced against the benefits of using mesh.

\section{Competing Interests}

The authors declare that they have no competing interests

\section{References}

1. Wu JM, Kawasaki A, Hundley AF, Dieter AA, Myers ER, et al. (2011) Predicting the number of women who will undergo incontinence and prolapse surgery, 2010 to 2050. Am J Obstet Gynecol 205: e1-230.e5.

2. Dandolu V, Akiyama M, Allenback G, Pathak P (2017) Mesh complications and failure rates after transvaginal mesh repair compared with abdominal or laparoscopic sacrocolpopexy and to native tissue repair in treating apical prolapse. Int Urogynecol J 28: 215-222.

3. Gaines N, Gupta P, Sirls LT (2016) Pelvic prolapse repair in the Era of Mesh. Curr Urol Rep 17: 20.

4. Maher C, Feiner B, Baessler K, Christmann-Schmid C, Haya N et al. (2016) Transvaginal mesh or grafts compared with native tissue repair for vaginal prolapse. Cochrane Database Syst Rev 2: CD012079.

5. Barski D, Otto T, Gerullis H (2014) Systematic review and classification of complications after anterior, posterior, apical, and total vaginal mesh implantation for prolapse repair. Surg Technol Int 24: 217-24.

6. Gutman RE (2016) Does the uterus need to be removed to correct uterovaginal prolapse? Curr Opin Obstet Gynecol 28: 435-440.

7. Maher C, Feiner B, Baessler K, Christmann-Schmid C, Haya N, et al. (2016) Surgery for women with apical vaginal prolapse. Cochrane Database Syst Rev 10: CD012376.

8. Maher C, Feiner B, Baessler K, Christmann-Schmid C, Haya N, et al (2016) Surgery for women with anterior compartment prolapse. Cochrane Database Syst Rev 11: CD004014.

9. Glazener CM, Breeman S, Elders A, Hemming C, Cooper KG, et al. (2017) Mesh, graft, or standard repair for women having primary transvaginal anterior or posterior compartment prolapse surgery: two parallel-group multicentre, randomised, controlled trials (PROSPECT). Lancet 389: 381 392. 